\title{
Citações de Obras de Bandura em Artigos de Periódicos de Psicologia Brasileiros: Uma Análise Preliminar
}

\author{
Roberta Gurgel Azzi ${ }^{1}$ \\ ${ }^{1}$ Universidade Estadual de Campinas, SP, Brasil. \\ Luiza Cristina Mauad Ferreira $^{1}$ \\ ${ }^{1}$ Universidade Estadual de Campinas, SP, Brasil. \\ Mônica Helena Tieppo Alves Gianfaldoni ${ }^{3}$ \\ ${ }^{3}$ Pontifícia Universidade Católica de São Paulo, SP, Brasil. \\ Ana Paula Basqueira ${ }^{2}$ \\ ${ }^{2}$ Anhanguera Educacional, SP, Brasil. \\ Maria do Carmo Guedes ${ }^{3}$ \\ ${ }^{3}$ Pontificia Universidade Católica de São Paulo, SP, Brasil. \\ asil.
}

Resumo: Para que seja possível a análise de uma produção científica por meio de citações e fontes bibliográficas é necessário um conjunto relevante de modelos quantitativos que permitam medir, estudar e analisar esse corpus teórico. A avaliação histórica da produção científica contextualiza e permite detectar o estado da arte e elaborar o planejamento de novas ações. Este artigo tem como objetivo descrever a recepção da teoria social cognitiva de Albert Bandura na historiografia da Psicologia no Brasil, apresentando resultados iniciais de levantamento realizado em periódicos científicos brasileiros. A partir da lista de periódicos disponíveis no sistema PePSIC na BVS-PSI, tomou-se como critério definidor a seleção de revistas que tivessem ao menos 10 exemplares publicados para análise. Vinte e oito periódicos científicos da área da Psicologia foram localizados, sendo 13 gerais e 15 direcionados a diferentes áreas de aplicação e, assim, um total de 848 exemplares de revistas científicas foi consultado. Embora não tenha sido localizado nenhum estudo semelhante a este, em outros países, que possa servir como referência para comparar os resultados apresentados, podemos dizer que, considerando a trajetória de reconhecimento internacional de contribuição do autor, sua inserção na forma de citações em periódicos brasileiros no período levantado não é expressiva. Análises posteriores estão sendo conduzidas para se construir um completo panorama das condições em que o pensamento banduriano foi abordado nos artigos de periódicos brasileiros.

Palavras-chave: Albert Bandura, Teoria Social Cognitiva, Análise de citações.

\section{Citations of Bandura's Works in Articles of Brazilian Psychology Journals: a Preliminary Analysis}

\begin{abstract}
In order to be able to analyze a scientific production through citations and bibliographical sources, a relevant set of quantitative models is necessary to measure, study and analyze them. The historical evaluation of the scientific production contextualizes the scenario and allows to detect the state of the art and the planning of new actions. In an attempt to find evidence of the insertion of Albert Bandura's in the historiography of Psychology in Brazil, this article presents initial results of a survey conducted in Brazilian scientific journals. From the list of journals available in the PePSIC system in the BVS-PSI, the journals with at least 10 published copies were selected for analysis. Ttwenty-eight scientific journals of the area of Psychology were found. A total of 848 copies of scientific journals were consulted. From these, some were about general issues and others aimed at different areas of application. Although no study similar to this one had been found in other countries, which could be used as a reference for comparing the presented results, we can say that considering the international recognition of the author's contribution, his insertion in the form of citations in Brazilian periodicals in the period covered is not significant. Further analyses are being conducted to construct a complete panorama of the conditions in which the Bandurian thought was approached in the articles of Brazilian journals.
\end{abstract}

Keywords: Albert Bandura, Social Cognitive Theory, Analysis of citations. 


\title{
Citas de Obras de Bandura en Artículos de Periódicos de Psicología Brasileños: Un Análisis Preliminar
}

\begin{abstract}
Resumen: Para que sea posible el análisis de una producción científica por medio de citas y fuentes bibliográficas es necesario un conjunto relevante de modelos cuantitativos que permitan medir, estudiar y analizar este corpus teórico. La evaluación histórica de la producción científica contextualiza y permite detectar su estado de arte y la planificación de nuevas acciones. Este artículo tiene como objetivo describir la recepción de la teoría social cognitiva de Albert Bandura en la historiografía de la Psicología en Brasil, presentando los resultados iniciales de una encuesta realizada en periódicos científicos brasileños. De la lista de periódicos disponibles en el sistema PePSIC en el BVS-PSI, el criterio de definición fue la selección de periódicos y revistas que tuviesen al menos 10 copias publicadas para su análisis. Se ubicaron 28 periódicos científicos de Psicología, siendo 13 generales y 15 dirigidas a diferentes áreas de aplicación y, por lo tanto, se consultó un total de 848 copias de revistas científicas. Aunque no se ha encontrado un estudio similar en otros países que pueda servir como referencia para comparar los resultados presentados, podemos decir que, considerando la trayectoria de reconocimiento internacional del autor, su inserción en forma de citas en revistas brasileñas en el período planteado no es expresivo. Se están realizando análisis posteriores para construir una visión general completa de las condiciones bajo las cuales se ha abordado el pensamiento banduriano en artículos de periódicos brasileños.
\end{abstract}

Palabras clave: Albert Bandura, Teoría Social Cognitiva, Análisis de citas.

Nenhuma produção científica está independente dos efeitos que ela produz, nem da acolhida que recebe. De fato, a historicidade de uma obra não se mede unicamente pela sua existência: ela reside na interação que exerce entre a obra e o mundo científico no qual se instala e com a qual evolui (Parrat-Dayan, 2008, p. 149).

Para que seja possível a análise de uma produção científica por meio de citações e fontes bibliográficas é necessário um conjunto relevante de modelos quantitativos que permitam medi-la, estudá-la e analisá-la. Narin (1994) afirma que sem o uso de indicadores quantitativos seria impossível entender todas as implicações do desenvolvimento científico e tecnológico contemporâneo. Esses indicadores possibilitam identificar uma série de relações e tendências, usuários, mensurar a disseminação da informação, e averiguar a abrangência de periódicos e autores. A análise da produção científica possibilita a avaliação de como tem sido a interlocução desta com os demais pesquisadores da área, entre os diferentes tipos de pesquisa se básica ou aplicada e ainda a elucidação de questões presentes nos dias de hoje e não a mera quantifica- ção (Ferreira, Guedes, \& Azzi, 2004). A avaliação histórica da produção científica contextualizada, permite detectar seu estado da arte e o planejamento de novas ações. No presente estudo, para cumprir o objetivo proposto, será tomado como referência o trabalho de Brozek (1998), quando considera o caminho da verificação quantitativa e a frequência de citações de trabalhos de autores como metodologia objetiva para se avaliar o impacto de determinadas obras. Investigar citações em periódicos é uma alternativa que favorece perceber a disseminação de perspectivas teóricas uma vez que, os periódicos foram idealizados também para agilizar o processo de comunicação científica. Por meio da análise de citações é possível verificar a contribuição que os autores têm oferecido a outras publicações em diferentes áreas como também verificar as tendências e direções das pesquisas publicadas e a contribuição efetiva destas (Ferreira 2003). Partindo dessas premissas é que essa pesquisa foi conduzida tendo como objetivo descrever a recepção da teoria social cognitiva de Albert Bandura na historiografia da Psicologia no Brasil, apresentando resultados iniciais de levantamento realizado em periódicos científicos brasileiros. Estes foram consultados para 
responder indagação a respeito de como, onde e quais os temas do pensamento do psicólogo Albert Bandura se apresentam em artigos de revistas brasileiras. Cabe destacar que os periódicos embasam uma análise que integra estudo mais amplo ${ }^{1}$ que explora os caminhos de inserção do pensamento banduriano em contexto brasileiro, sendo parte de projeto que problematiza as divergências da Teoria Social Cognitiva com a Análise do Comportamento, a partir de posicionamento de críticos à primeira proposta mencionada.

Ao longo de sua trajetória acadêmica, Bandura (nascido em 1925, em Alberta no Canadá) desenvolveu a denominada "Teoria Social Cognitiva", atualmente entre as teorias gerais de Psicologia, que tem sido contemplada em diferentes áreas do conhecimento. Essa diversidade revela um dos aspectos da importância de sua contribuição enquanto um dos autores no campo da Psicologia que tem suas ideias destacadas (Azzi, 2014). Pode-se inicialmente considerar que no Monitor on Psychology da American Psychology Associations (APA) de julho/agosto de 2002 já se verificava duas referências a ele.

\section{Psychologists were put to a popularity contest in a new study that appears in the Review of General Psychology (vol.6, n. 2), which ranks 99 of the 100 most eminent psychologists of the 20th century.}

\section{B. F. Skinner topped the list, followed by Jean} Piaget, Sigmund Freud and Albert Bandura (Dittmann, 2002, p. 28).

Cabe destacar que Diener, Oishi e Park (2014), partindo da combinação de três critérios de análise (a) reconhecimento: identificação da inclusão das ideias do autor em livros-texto; (b) impacto: coletado a partir de citações do autor; e (c) respeito: considerando o número de premiações, construíram a lista dos 100 eminentes psicólogos da Era Moderna com carreiras de destaque após $1956^{2}$, na qual Bandura figura em primeiro lugar. Outra evidência da importância desse autor pode ser encontrada na análise das publicações mais citadas nas Ciências Sociais, de acordo com consulta no Google Scholar, realizada por Elliott Green este ano (Green, 2016). Segundo seus achados, o livro Social foundations of thought and action: A Social Cognitive Theory, publicado em 1986, e o artigo Self-efficay, de 1977, constam de listas sobre os mais citados na literatura de Ciências Sociais. Excluindo artigos de metodologia, o artigo Self-efficacy é o terceiro dentre os 10 artigos mais citados.

Sua produção intelectual é extensa, como pode ser verificado nas tabelas construídas e apresentadas ao longo do texto e, é composta por nove livros, listados na Tabela 1. Os livros revelam um trabalho mais sistematizado de organização das ideias e estudos empíricos e serão, neste artigo, tomados como elementos referenciais de sua obra ao longo do tempo da construção da Teoria Social Cognitiva (TSC). Pode-se perceber que Bandura teve como parceiro Richard Walters em seus dois primeiros livros; os demais foram escritos individualmente e assim como os demais tiveram traduções para diversos idiomas como: espanhol, russo, japonês, francês, alemão, italiano, chinês e coreano.

Não consta na Tabela 1, mas, além da produção de textos autorais, Bandura editou, sozinho ou com parceiros, mais quatro livros, sendo o de 2008 publicado em português:

1. Bandura, A. (1971). Analysis of modeling processes. In A. Bandura (Ed.), Psychological modeling: Conflicting theories. Chicago: Aldine-Atherton.

2. Bandura, A., \& Ribes-Inesta, E. (Eds.) (1976). Analysis of delinquency and aggression. Hillsdale, NJ: Erlbaum.

3. Bandura, A. (Ed.). (1995). Self-efficacy in changing societies. Cambridge: Cambridge University Press.

4. Bandura, A., Azzi, R. G., Polydoro, S.A.J. (Eds). (2008). Terra social cognitiva: Conceitos básicos. Porto Alegre, RS: Artmed.

A Tabela 2 mostra a distribuição de suas obras ao longo dos anos em lista que foi fornecida pelo próprio autor em 2016. Os dados sobre a natureza da produção, ou seja, livros, artigos, capítulo de livros, verbetes para enciclopédia e textos de anais de congresso, foram divididos em períodos, tomando-se como referência os anos de publicação dos livros em cada período. Analisando-se cada um deles, percebe-se o predomínio de artigos em todos os perío-

\footnotetext{
${ }^{1}$ O projeto ora apresentado está relacionado ao projeto “Teoria social cognitiva: divergências teóricas com a análise do comportamento e inserção no cenário brasileiro", coordenado pela proponente e que está em desenvolvimento com apoio da Fundação de Amparo à Pesquisa do Estado de S. Paulo - FAPESP (Processo n ${ }^{\circ}$ 2015/15211-2), com a colaboração de Maria do Carmo Guedes, Luiza Cristina Mauad Ferreira, Mônica Helena Tieppo Alves Gianfaldoni e Ana Paula Basqueira.

${ }^{2}$ Primeiro ano do prêmio de distinção da American Psychological Association (APA).
} 
Tabela 1

Livros escritos por Bandura ao longo do desenvolvimento de suas perspectivas teóricas por ano.

\begin{tabular}{lc}
\hline Ano & Livro \\
\hline 1959 & Adolescent aggression, com Richard H. Walters \\
1963 & Social learning and personality development, com Richard H. Walters \\
1969 & Principles of behavior modification - o único livro traduzido para o português e publicado no Brasil em 1979 \\
1971 & Social learning theory \\
1973 & Aggression: A social learning analysis \\
1977 & Social learning theory- nova versão \\
1986 & Self-efficacy: The exercise of control \\
1997 & Moral Disengagement \\
2016 & Solions of thought and action: social cognitive theory \\
\hline
\end{tabular}

Fonte: Lista de publicações enviada pelo autor em março de 2016.

dos analisados sendo que, nos últimos períodos, há aumento no número de capítulos e de outras produções. Pode-se observar, a partir dos dados apresentados na Tabela 2, como esta produção se revela em termos de natureza de sua obra. Artigos são a maioria em sua produção (180), seguidos de capítulos de livros (79). Bandura produziu, ainda, uma série de outros materiais que incluem verbetes em enciclopédias e textos em anais de congressos (50) apontados na categoria Outros, na Tabela 2.

Pressupõem-se que a obra de Bandura foi introduzida no Brasil a partir o final da década de 1970 porque um de seus livros - Principles of behavior modification - foi traduzido para o português e publicado aqui dez anos após sua edição original que é de 1969. Entretanto, parte-se neste estudo da hipótese que o movimento de recepção das ideias de Bandura não aconteceu no Brasil, até recentemente, diferentemente de outros países que o contemplaram com prêmios (professor emérito da: University of British Columbia, 1979; University of Lethbridge, 1983; University of New Brunswick, 1985; State University of New York, Stony Brook, 1987; University of Waterloo, 1990; Freie Universitat Berlin, 1990; University of Salamanca, 1992; Indiana University, 1993; University of Rome, "La Sapienza”, 1994; Leiden University, 1995;

Tabela 2

Trabalhos acadêmicos de Bandura ao longo do tempo, de acordo com a natureza da produção.

\begin{tabular}{lcccccccc}
\hline Período & SI $^{*}$ & Artigos & Livros & Capítulo & Outros & N Total & $\%$ & \% acumulada \\
\hline $1953-1958$ & 1 & 6 & - & - & - & 7 & $2,16 \%$ & \\
$1959-1962$ & - & 6 & 1 & 1 & 1 & 9 & $2,77 \%$ & $4,93 \%$ \\
$1963-1968$ & - & 20 & 1 & 5 & 4 & 30 & $9,25 \%$ & $14,18 \%$ \\
$1969-1972$ & - & 4 & $2^{* *}$ & 5 & 2 & 13 & $4,01 \%$ & $18,19 \%$ \\
$1973-1976$ & 1 & 14 & 1 & 5 & 1 & 22 & $6,79 \%$ & $24,98 \%$ \\
$1977-1985$ & 4 & 29 & 1 & 6 & 1 & 41 & $12,65 \%$ & $37,63 \%$ \\
$1986-1996$ & - & 39 & 1 & 22 & 10 & 72 & $22,22 \%$ & $59,85 \%$ \\
$1997-2015$ & - & 62 & 1 & 35 & 31 & 129 & $39,81 \%$ & $99,66 \%$ \\
2016 & - & - & 1 & - & - & 1 & $0,31 \%$ & $99,97 \%$ \\
\hline Total & 6 & 180 & 9 & 79 & 50 & 324 & $100,00 \%$ &
\end{tabular}

*Sem informação suficiente para identificação da natureza da produção.

**Um texto preliminar sobre a teoria foi contabilizado como livro (texto de 1971), mas não contado separado, pois ele foi posteriormente ampliado e publicado em 1977. Dado que um corte de período já estava marcado para 1969, decidiu-se manter os dois no mesmo período, para não produzir mais um período, que seria muito curto. 
Alfred University, 1995; Pennsylvania State University, 1999; Universitat Jaume, 2002; Graduate Center of the City University of New York, 2002; University of Athens, 2003; University of Catania, 2004; Universidad del Valle de Guatemala, 2009; University of Alberta, 2010; University of Ottawa, Canadá, 2013); traduções de obras e homenagens derivadas de sua obra (Bandura http://p20motivationlab.org/acessado em abril 2018).

Nesse sentido, as questões que norteiam o estudo mais amplo, do qual este recorte faz parte podem ser assim enunciadas: seria verdade que as ideias de Bandura demoraram a ser contempladas no Brasil? Se sim, quais razões poderiam explicar a suposta demora da apropriação das ideias do autor em cenário brasileiro? Baixa relevância e aplicabilidade da teoria formulada? Se sim, por que o movimento em outros países parece ter acontecido de forma diversa? Seria a barreira da língua inglesa um limitador? Quais outras condições podem ter contribuído para esta situação? Teria a adesão a esta perspectiva passado a ser significativa após sua formulação mais integrada como apresentada no livro Social Foundations of Thought and Action publicado em1986? Teria sido a presença forte de outras abordagens exploradas nos cursos de formação um impeditivo para a divulgação da teoria social cognitiva? Estas questões sinalizam hipóteses não excludentes, mas que parecem merecedoras de verificação, com destaque para a formulação integrada da teoria e a da concorrência com outras abordagens psicológicas difundidas no Brasil.

Neste artigo, discutem-se as informações relacionadas à primeira questão e às que dela derivam: as ideias de Bandura estão presentes nas publicações científicas no Brasil? Se estão, a partir de que período começam a ter maior visibilidade? Há diferenças na recepção de suas ideias em função das áreas da Psicologia?

\section{Método}

Com o intuito de investigar as questões assinaladas, este trabalho foi delineado com a estratégia de busca de evidências que as refutem ou as corroborem. A partir da lista de 111 periódicos disponíveis no sistema PePSIC na BVS-PSI à época, foram localizados 28 periódicos científicos da área da Psicologia que tivessem ao menos 10 exemplares publicados para análise, sendo 13 de aspectos gerais da Psicologia e 15 direcionados a diferentes áreas de aplicação.
Essa primeira organização do material feita de forma a permitir posteriores análises sobre áreas da Psicologia com maior inserção de Bandura, permitiu distribuir os periódicos em dois grandes grupamentos: 1) Geral, em que são tratados vários temas, assuntos e diferentes abordagens psicológicas e 2) Específico, em que agrupem-se os temas específicos em: Educação, Saúde, Carreira e Trabalho, Avaliação, Política e Comportamento. A inclusão das revistas em cada uma dessas categorias foi norteada pelas descrições dos perfis nos próprios periódicos e pelo conhecimento das pesquisadoras.

Na Tabela 3 encontra-se a lista, por grupamento, dos 28 periódicos analisados, o período de publicações, a indicação do último exemplar consultado e o total de exemplares consultados.

A Tabela 3 indica qual foi o corpus selecionado e utilizado para responder às questões desta pesquisa; assim, um total de 848 exemplares de revistas científicas foi consultado. A inspeção visual da tabela permite verificar que, embora o número de revistas por agrupamento tenha variado entre as especificidades de suas áreas de inserção, todos os periódicos analisados ultrapassaram o critério mínimo de 10 números publicados, alguns com um volume de exemplares que revela longa existência da publicação, como pode ser visto na coluna relativa ao período analisado.

Considerando o total de 848 exemplares consultados dentre os 28 periódicos privilegiados por este levantamento abrangendo o período de 1979-2015, acredita-se que as informações obtidas são representativas da publicação científica em forma de artigos e podem servir de balizamento para responder às perguntas formuladas para o presente texto.

\section{Resultados e Discussão}

A Tabela 4 apresenta o número de artigos com citações a obras de Bandura, por periódico. Essas informações permitem identificar a proporção de artigos com citações em relação ao número de exemplares consultados e, ainda, qual o periódico e a que agrupamento pertence.

Nota-se que, considerando a proporção entre o número de artigos com citações por número de exemplares de cada periódico consultado, três revistas apresentam uma relação acima de 0,5 , ou seja, mais da metade dos números publicados apresenta alguma citação de Bandura. São elas, em ordem decrescente, 
Tabela 3

Periódicos por grupamentos, períodos e exemplares consultados na pesquisa.

\begin{tabular}{|c|c|c|c|}
\hline Periódico & Período & $\begin{array}{c}\text { Último } \\
\text { volume } \\
\text { consultado }\end{array}$ & $\begin{array}{l}\text { Número de } \\
\text { exemplares } \\
\text { consultados }\end{array}$ \\
\hline \multicolumn{4}{|l|}{ GERAIS } \\
\hline Arquivos Brasileiros de Psicologia & $2003 / 2015$ & $2015 / 2$ & 30 \\
\hline Boletim de Psicologia & $2005 / 2014$ & $2014 / 140$ & 18 \\
\hline Estudos e Pesquisas em Psicologia & $2003 / 2015$ & $2015 / 2$ & 33 \\
\hline Estudos Interdisciplinares em Psicologia & $2010 / 2014$ & $2014 / 2$ & 10 \\
\hline Revista Interinstitucional de Psicologia & $2010 / 2014$ & $2014 / 2$ & 15 \\
\hline Psic: Revista da Vetor Editora & $2002 / 2008$ & 2008 & 14 \\
\hline Psicologia Ciência e Profissão & $1979 / 2015$ & $2015 / 4$ & 103 \\
\hline Psicologia em Pesquisa & $2007 / 2014$ & $2014 / 1$ & 15 \\
\hline Psicologia em Revista & $2005 / 2014$ & $2014 / 2$ & 25 \\
\hline Psicologia Teoria e Prática & $2002 / 2015$ & $2015 / 1$ & 34 \\
\hline Psicologia USP & $1990 / 2005$ & $2015 / 3$ & 48 \\
\hline Psico-USF & $2001 / 2015$ & $2015 / 3$ & 35 \\
\hline Temas em Psicologia & $1993 / 2015$ & $2015 / 4$ & 61 \\
\hline \multicolumn{4}{|l|}{ EDUCAÇÃO } \\
\hline Psicologia da Educação & $2004 / 2015$ & $2015 / 40$ & 22 \\
\hline Psicologia Escolar e Educacional & $1996 / 2015$ & $2015 / 3$ & 42 \\
\hline Revista Psicopedagogia & $2006 / 2015$ & $2015 / 98$ & 34 \\
\hline \multicolumn{4}{|l|}{ SAÚDE } \\
\hline Psicologia Hospitalar & $2004 / 2014$ & $2014 / 2$ & 21 \\
\hline Revista da SBPH & $2004 / 2014$ & $2014 / 1$ & 21 \\
\hline SMAD Revista Eletrônica Saúde Mental Álcool e Drogas & $2005 / 2015$ & $2015 / 3$ & 28 \\
\hline \multicolumn{4}{|l|}{ CARREIRA/TRABALHO } \\
\hline Cadernos de Psicologia Social do Trabalho & $1998 / 2014$ & $2014 / 3$ & 28 \\
\hline Revista Brasileira de Orientação Profissional & $2003 / 2015$ & $2015 / 1$ & 24 \\
\hline Revista Psicologia Organizações e Trabalho & $2001 / 2015$ & $2015 / 4$ & 36 \\
\hline \multicolumn{4}{|l|}{ AVALIAÇÃO } \\
\hline Avaliação Psicológica & $2002 / 2015$ & $2015 / 2$ & 35 \\
\hline \multicolumn{4}{|l|}{ POLÍTICA } \\
\hline Revista Psicologia Política & $2007 / 2014$ & $2014 / 30$ & 17 \\
\hline \multicolumn{4}{|l|}{ COMPORTAMENTO } \\
\hline Acta Comportamentalia & $2006 / 2015$ & $2015 / 1$ & 23 \\
\hline Ciências \& Cognição & $2004 / 2013$ & $2013 / 1$ & 25 \\
\hline Revista Brasileira de Terapia Comportamental e Cognitiva & $1999 / 2014$ & $2014 / 1$ & 33 \\
\hline Revista Brasileira de Terapias Cognitivas & $2005 / 2013$ & $2013 / 2$ & 18 \\
\hline TOTAL & & & 848 \\
\hline
\end{tabular}


Tabela 4

Número de artigos com citações a obras de Bandura, por exemplares consultados nos periódicos analisados, de acordo com o agrupamento e título do periódico.

\begin{tabular}{|c|c|c|c|}
\hline Periódico & $\begin{array}{l}\text { Número de } \\
\text { exemplares } \\
\text { consultados }\end{array}$ & $\begin{array}{l}\text { Número de } \\
\text { artigos com } \\
\text { citações }\end{array}$ & $\begin{array}{c}\text { Proporção } \\
\text { Exemplares/citações }\end{array}$ \\
\hline GERAIS & 441 & 72 & 0,16 \\
\hline Arquivos Brasileiros de Psicologia & 30 & 2 & 0,06 \\
\hline Boletim de Psicologia & 18 & 2 & 0,11 \\
\hline Estudos e Pesquisas em Psicologia & 33 & 6 & 0,18 \\
\hline Estudos Interdisciplinares em Psicologia & 10 & 1 & 0,10 \\
\hline Revista Interinstitucional de Psicologia & 15 & 1 & 0,06 \\
\hline Psic: Revista da Vetor Editora & 14 & 0 & 0,00 \\
\hline Psicologia Ciência e Profissão & 103 & 10 & 0,09 \\
\hline Psicologia em Pesquisa & 15 & 4 & 0,26 \\
\hline Psicologia em Revista & 25 & 1 & 0,04 \\
\hline Psicologia Teoria e Prática & 34 & 9 & 0,26 \\
\hline Psicologia USP & 48 & 3 & 0,06 \\
\hline Psico-USF & 35 & 18 & 0,51 \\
\hline Temas em Psicologia & 61 & 15 & 0,24 \\
\hline EDUCAÇÃO & 98 & 16 & 0,16 \\
\hline Psicologia da educação & 22 & 3 & 0,13 \\
\hline Psicologia Escolar e Educacional & 42 & 11 & 0,26 \\
\hline Revista Psicopedagogia & 34 & 2 & 0,05 \\
\hline SAÚDE & 70 & 2 & 0,02 \\
\hline Psicologia Hospitalar & 21 & 0 & 0,00 \\
\hline Revista da SBPH & 21 & 1 & 0,04 \\
\hline SMAD Revista Eletrônica Saúde Mental Álcool e Drogas & 28 & 1 & 0,03 \\
\hline CARREIRA/TRABALHO & 88 & 28 & $\mathbf{0 , 3 1}$ \\
\hline Cadernos de Psicologia Social do Trabalho & 28 & 0 & 0,00 \\
\hline Revista Brasileira de Orientação Profissional & 24 & 21 & 0,87 \\
\hline Revista Psicologia Organizações e Trabalho & 36 & 7 & 0,19 \\
\hline AVALIAÇÃO & 35 & 24 & 0,68 \\
\hline Avaliação Psicológica & 35 & 24 & 0,68 \\
\hline POLÍTICA & 17 & 2 & 0,11 \\
\hline Revista Psicologia Política & 17 & 2 & 0,11 \\
\hline COMPORTAMENTO & 99 & 20 & $\mathbf{0 , 2 0}$ \\
\hline Acta Comportamentalia & 23 & 4 & 0,17 \\
\hline Ciências \& Cognição & 25 & 2 & 0,08 \\
\hline Revista Brasileira de Terapia Comportamental e Cognitiva & 33 & 6 & 0,18 \\
\hline Revista Brasileira de Terapias Cognitivas & 18 & 8 & 0,44 \\
\hline TOTAL & 848 & 164 & 0,19 \\
\hline
\end{tabular}


Revista Brasileira de Orientação Profissional $(0,87)$, Avaliação Psicológica $(0,68)$ e Psico-USF $(0,51)$. Uma particularidade que emerge destes três periódicos é que pesquisadores portugueses difundem as ideias de Bandura em publicações brasileiras. Vários artigos dentre aqueles em que se encontram citações de Bandura, são de colegas de universidades portuguesas, país em que a presença das ideias de Bandura encontra-se mais disseminada, pelo menos nas áreas de orientação profissional e educação. Esta parece uma trilha a ser perseguida posteriormente, em novos estudos de exploração aprofundada dos resultados aqui apresentados.

Na Tabela 5 apresenta-se o número de artigos com citações de Bandura por ano e área dos periódicos analisados.
Embora não tenha sido localizado nenhum estudo semelhante a este, em outros países, que possa servir como referência para comparar os resultados apresentados nas Tabelas 4 e 5, pode-se dizer que, considerando a trajetória de reconhecimento internacional de contribuição do autor, sua inserção na forma de citações em periódicos brasileiros no período levantado não é expressiva. Como já mencionado, foram identificadas citações a alguma obra de Bandura em 167 artigos de 848 exemplares de periódicos consultados via portal PePSIC- BVS, ou seja, uma proporção pouco expressiva de 0,16 foi estabelecida. Os dados apresentados até o momento permitem afirmar que, no critério de frequência de citações utilizado como evidência, há uma baixa inserção das ideias bandurianas no cenário de discussões e investigações dos

\section{Tabela 5}

Número de artigos com citações de obras de Bandura, por ano e área dos periódicos analisados.

\begin{tabular}{|c|c|c|c|c|c|c|c|c|}
\hline $\begin{array}{l}\text { Ano do } \\
\text { artigo/área }\end{array}$ & Gerais & Educação & Saúde & $\begin{array}{c}\text { Carreira e } \\
\text { trabalho }\end{array}$ & Política & Avaliação & $\begin{array}{l}\text { Compor- } \\
\text { tamento }\end{array}$ & Total \\
\hline 1981 & 1 & - & - & - & - & - & - & 1 \\
\hline 1982 & - & - & - & - & - & - & - & \\
\hline 1983 & 1 & - & - & - & - & - & - & 1 \\
\hline $1984 \ldots$ & - & - & - & - & - & - & - & - \\
\hline ...1997 & - & - & - & - & - & - & - & - \\
\hline 1998 & 1 & - & - & - & - & - & - & 1 \\
\hline 1999 & - & - & - & - & - & - & 2 & 2 \\
\hline 2000 & - & - & - & - & - & - & - & \\
\hline 2001 & 1 & - & - & - & - & - & - & 1 \\
\hline 2002 & 1 & 1 & & 1 & & & & 3 \\
\hline 2003 & 1 & 1 & & & & 1 & 1 & 4 \\
\hline 2004 & 2 & & & 2 & & & & 4 \\
\hline 2005 & 5 & 1 & & & & 2 & 1 & 9 \\
\hline 2006 & 1 & 1 & & 1 & & 2 & 4 & 9 \\
\hline 2007 & 4 & & & & & 1 & 2 & 7 \\
\hline 2008 & 4 & 4 & & 3 & & 2 & & 13 \\
\hline 2009 & 5 & 3 & 1 & & & 1 & 1 & 11 \\
\hline 2010 & 3 & & & 3 & 1 & 2 & 2 & 11 \\
\hline 2011 & 18 & 2 & & 3 & & 1 & 5 & 29 \\
\hline 2012 & 7 & & & 3 & & 3 & & 13 \\
\hline 2013 & 3 & & & 4 & 1 & & 1 & 9 \\
\hline 2014 & 8 & 3 & & 3 & & 5 & 1 & 20 \\
\hline 2015 & 6 & & 1 & 5 & & 4 & & 16 \\
\hline Total & 72 & 16 & 2 & 28 & 2 & 24 & 20 & 164 \\
\hline
\end{tabular}


pesquisadores brasileiros, em movimento interno de divulgação científica de seus estudos. Se efetuada a contagem do número de artigos por exemplares consultados, esta medida destacaria ainda mais a informação que sustenta afirmar essa baixa inserção das ideias de Bandura no cenário da Psicologia no Brasil.

Nota-se que, até 1998, apenas revistas categorizadas no grupamento de temas gerais chegam a citar Bandura. A partir de 2002, esta presença vai em um crescente e começa a ser registrada nas revistas por área e assunto, aumentando o número de citações observadas a cada ano sendo que nos anos de 2011 e 2014 verifica-se mais de 20 artigos com citações. Se confrontar-se com o dado de que é a partir de 2001 que se revela a presença do pensamento banduriano nos periódicos brasileiros, com maior frequência por ano, com os dados da Tabela 2, vê-se que, por volta de 2001, quase $70 \%$ da obra de Bandura já estava publicada. Esta conclusão pode ser considerada uma evidência de que, de fato, o pensamento banduriano chegou tardiamente ao cenário da divulgação científica representada por periódicos brasileiros.

Se a análise for feita por áreas, excetuando-se os artigos publicados em revistas gerais (72), percebe-se que Carreira e Trabalho (28), Avaliação Psicológica (24) e Comportamentalismo (21) são as que publicaram mais artigos citando Bandura e o referenciando. Uma análise mais apurada sobre onde o pensamento de Bandura está mais disseminado já está sendo realizada como próximas etapas do projeto. Como ensaio do prosseguimento dessa investigação pode-se apresentar uma primeira análise qualitativa realizada que contempla os cinco primeiros artigos, anteriores a 2000, em que há referências a trabalhos de Bandura. Na Tabela 6 está apresentada a organização das informações coletadas sobre os cinco artigos. Estes foram publicados em três revistas, sendo duas do grupamento Geral - Psicologia Ciência e Profissão e Temas em Psicologia - e uma do grupamento Específico Revista Brasileira de Terapia Comportamental e Cognitiva. A tabela 6 como organizadora inicial, apresenta o ano de publicação do artigo, nome do artigo, nome da revista, autor(es), ano de publicação e número de referências a Bandura.

A Tabela 7 apresenta cada artigo encontrado antes de 2000 com citação a trabalhos de Bandura, indica qual foi o trecho em que a menção a este autor foi feita e o número de textos de Bandura citados.

\section{Tabela 6}

Organização das informações coletadas sobre cinco artigos.

\begin{tabular}{|c|c|c|c|c|}
\hline $\begin{array}{l}\text { Ano do } \\
\text { artigo }\end{array}$ & Gerais & Periódicos gerais & Periódicos específicos/comportamento & Total \\
\hline 1981 & 1 & $\begin{array}{l}\text { Aprendizagem por observação: } \\
\text { perspectivas teóricas e contribuições } \\
\text { para o planejamento instrucional } \\
\text { Jairo Eduardo Borges-Andrade } \\
\text { Psicologia, Ciência e Profissão, v1, n2 }\end{array}$ & - & 1 \\
\hline 1983 & 1 & $\begin{array}{l}\text { Terapia comportamental cognitiva: uma } \\
\text { comparação entre perspectivas } \\
\text { Rachel Rodrigues Kerbauy } \\
\text { Psicologia, Ciência e Profissão, v3, n2 }\end{array}$ & - & 1 \\
\hline 1998 & 1 & $\begin{array}{l}\text { A religião como fenômeno psicológico } \\
\text { Franco Lo Prestí Seminério } \\
\text { Temas em Psicologia, v6, n2 }\end{array}$ & - & 1 \\
\hline 1999 & - & - & $\begin{array}{l}\text { Cognição: contato com contingências e regras } \\
\text { Lorismário Ernesto Simonassi } \\
\text { Revista Brasileira de Terapia } \\
\text { Comportamental e Cognitiva, v1, n1 }\end{array}$ & 1 \\
\hline 1999 & - & - & $\begin{array}{l}\text { O que e um skinneriano? Uma reflexão sobre } \\
\text { mestres, discípulos e influência intelectual } \\
\text { Júlio César de Rose } \\
\text { Revista Brasileira de Terapia } \\
\text { Comportamental e Cognitiva, v1, n1 }\end{array}$ & 1 \\
\hline
\end{tabular}


Tabela 7

Trechos com menção a Bandura em cada artigo localizado anterior a 2000.

\begin{tabular}{lccc}
\hline $\begin{array}{l}\text { Ano do } \\
\text { artigo }\end{array}$ & $\begin{array}{c}\text { Referência do } \\
\text { artigo }\end{array}$ & Trechos em que Bandura é citado & $\begin{array}{c}\text { Número de } \\
\text { referências } \\
\text { de Bandura }\end{array}$ \\
\hline
\end{tabular}

Pág. 4 "O modelo é um ser humano ou é um objeto ou desenho que se assemelham com um ser humano (Bandura, 1977)".

Pág. 5 “Depois, Skinner (1953) explicou imitação delineando um paradigma de discriminação, provendo assim uma explanação S-R. Nos anos sessenta, Bandura levou a cabo um extensivo estudo sobre figuração, explicando a aquisição de respostas imitativas com um paradigma de reforçamento vicariante (Bandura, 1962; Bandura, \&Walters, 1963; Bandura, 1965)". "A concepção de aprendizagem social, tal como foi inicialmente descrita por Bandura (1969), explica figuração em termos de reforçamento vicariante”. Pág. 6 "Recentemente, Bandura (1977) alargou consideravelmente sua abordagem de aprendizagem por figuração. Bandura (1977) considera a aprendizagem por figuração como uma maneira menos árdua e menos arriscada de aprender do que a aprendizagem por consequências de respostas (reforçamento contingente)".

Pág. 7 "Três tipos de reforçadores são identificados: externo, vicariante e autorreforçamento. Estes três tipos de reforçadores teriam um papel antecipatório. Assim, eles são considerados importantes como fatores antecedentes e não como consequências. Todos os três tipos são 'considerados como condição facilitativa e não necessária...”' (Bandura, 1977, p.37).

Aprendizagem por observação: perspectivas teóricas e contribuições para o

1981 planejamento instrucional Jairo Eduardo Borges-Andrade Psicologia, Ciência e Profissão, v1, n2 Pág. 8 “Bandura (1977) estende esta afirmação para todos os tipos de resultados de aprendizagem: cognitivos, afetivos e psicomotores. Alguns exemplos do uso de figuração em psicoterapia são para a dessensibilização de fobias (Bandura, 1968)".

Pág. 14. "As crianças na condição de figuração aumentaram seu nível de interação social para o nível encontrado entre crianças sem problemas de retraimento social. Não houve mudanças entre as crianças do grupo controle. Ambos os estudos (Bandura e McDonald, 1963; O'Connor, 1969) ilustram como figuração pode ser usada para a aprendizagem de atitudes”. Pág. 15 "Bandura e Huston (1961) usaram duas condições de tratamento. Na condição de interação social os sujeitos tiveram um relacionamento socialmente recompensador com o modelo”.

Pág. 17 "Bandura, Ross e Ross (1961) verificaram que o comportamento do modelo masculino tinha maior influência do que o comportamento do modelo feminino no comportamento agressivo imitativo de crianças de ambos os sexos, em jardim de infância”.

Pág. 23 "Bandura e Huston (1961), que compararam interações sociais 'recompensadoras' e 'não-recompensadoras' entre modelo e sujeitos, também usaram habilidades motoras como variável dependente. Eles obtiveram mais reprodução imediata de respostas de 'marchar' com sujeitos sob condição de modelo que interagia de maneira socialmente 'recompensadora'. Bandura, Ross e Ross (1963) encontraram padrões de resultados similares com respostas motoras e atitudes: imitações imediatas foram mais frequentes quando o modelo foi um controlador do que quando o modelo foi um competidor por reforçadores”.

Pág. 45. "Nestes estudos, codificação verbal era entendida como a afirmação verbal da regra subjacente. Ao contrário das definições dadas por Bandura (1977, p. 39-40)”.

Pág. 50 "Mais três estudos aparentemente pretenderam comparar os efeitos de codificação verbal e de figuração por demonstração, no estabelecimento de comportamentos governados por regras (Bandura e Jeffery, 1973; Clarke, Manton, Viney e Hayes, 1975; Yoder e Forehand, 1974)”. 
Continuação

\begin{tabular}{|c|c|c|c|}
\hline $\begin{array}{l}\text { Ano do } \\
\text { artigo }\end{array}$ & $\begin{array}{l}\text { Referência do } \\
\text { artigo }\end{array}$ & Trechos em que Bandura é citado & $\begin{array}{l}\text { Número de } \\
\text { referências } \\
\text { de Bandura }\end{array}$ \\
\hline 1983 & $\begin{array}{l}\text { Terapia } \\
\text { comportamental } \\
\text { cognitiva: uma } \\
\text { comparação entre } \\
\text { perspectivas } \\
\text { Rachel Rodrigues } \\
\text { Kerbauy } \\
\text { Psicologia, } \\
\text { Ciência e } \\
\text { Profissão, v3, n2 }\end{array}$ & $\begin{array}{l}\text { Pág. } 3 \text { “Falando em TCC aceitam-se cognições e mediações. Passa-se a } \\
\text { aceitar cognições (percepções, pensamentos, crenças) como tendo uma } \\
\text { influência direta no comportamento (Bandura, 1969; Meichembaum, } \\
\text { 1974; Ellis, } 1966 \text { e Beck, 1963)”. }\end{array}$ & 1 \\
\hline
\end{tabular}

A religião como

fenômeno

psicológico

1998 Franco Lo Prestí

Seminério

Pág. 10 “O trabalho incansável de Bandura (1977) vem apontando o risco da difusão desses paradigmas. Quando o espírito vingativo é incentivado junto com a intolerância em qualquer área, encontramos o triste binômio

Temas em que leva à destrutividade".

Psicologia, v6, n2

Pág. 1. "Um certo número de teorias diferentes foi sugerido no que se refere ao papel funcional das atividades simbólicas, que geralmente é incluído no termo geral "consciência" no processo de modificação do comportamento (Bandura, 1979, p. 333)”.

"Os processos de modificação do comportamento são afetados pela consciência que a pessoa tem dos estímulos que a atingem, das respostas que exibem, das consequências dos seus comportamentos e das contingências que existem entre estes últimos eventos (Bandura, 1979, p. 333)".

Pág. 4. "Por exemplo, Bandura (1979) citou resultados de experimentos em que a descrição é condição necessária para aumento da frequência do

Cognição: contato com contingências e regras Lorismário Ernesto

1999 Simonassi comportamento, embora cite também experimentos em que a frequência da resposta aumentou independentemente da descrição. Bandura (1979) aponta que problemas metodológicos podem ser os responsáveis por tais resultados discrepantes. Entre os problemas, dois deles merecem atenção: 1. "A consciência (descrição) geralmente é tratada como um fenômeno de tudo ou nada $\sim 2$, quando de fato ela pode variar desde uma determinação correta por hipóteses parcialmente correlacionadas a noções altamente Revista Brasileira errôneas da razão pela qual o sujeito está sendo reforçado (p. 337) de Terapia 2. "A consciência é inferida das respostas a uma série de questões de Comportamental entrevista*2 progressivamente mais sugestivas. Portanto; o número e Cognitiva, $\mathrm{vl}, \mathrm{nl}$ de sujeitos julgados cônscios é determinado, em certa extensão, pelo número e natureza das pistas informativas veiculadas pela sondagem nas entrevistas. *2".

"Resultados empíricos permitem que se afirme que não. Os livros-textos contém experimentos que relatam tais tipos de resultados (cf. Catania, 1999; Bandura, 1979; Millenson,1979)".

Pág. 5. "Há, no entanto, na literatura (cf. Bandura, 1979) relatos indicativos de que 'as descrições das respostas exigidas para reforçamento, geralmente acusam um aumento substancial das respostas apropriadas'”. "O que pode ocorrer é que uma regra (descrição) facilite a solução do problema ou o aumento da frequência como citam Spielberger e De Nike (1966), no sentido de que a descrição faz com que o aumento seja abrupto como demonstraram Philbrick e Postman (citado em Bandura, 1979)". 
Continuação

\begin{tabular}{|c|c|c|c|}
\hline $\begin{array}{l}\text { Ano do } \\
\text { artigo }\end{array}$ & $\begin{array}{l}\text { Referência do } \\
\text { artigo }\end{array}$ & Trechos em que Bandura é citado & $\begin{array}{l}\text { Número de } \\
\text { referências } \\
\text { de Bandura }\end{array}$ \\
\hline & $\begin{array}{l}\text { O que é um } \\
\text { skinneriano? } \\
\text { Uma reflexão } \\
\text { sobre mestres, } \\
\text { discípulos } \\
\text { e influência } \\
\text { intelectual } \\
\text { Júlio César de } \\
\text { Rose } \\
\text { Revista Brasileira } \\
\text { de Terapia } \\
\text { Comportamental } \\
\text { e Cognitiva, vl, nl }\end{array}$ & $\begin{array}{l}\text { Pág. 4. "Talvez Bandura possa situar-se aí, no início de sua carreira, antes } \\
\text { que ele se tornasse mais decididamente cognitivista. Nesta fase inicial da } \\
\text { carreira, ele dedicou-se ao estudo de fenômenos da maior importância } \\
\text { para o comportamento humano (e.g., Bandura, 1965; Bandura, Ross } \\
\text { \& Ross, 1961), levando para o estudo destes fenômenos o rigor da } \\
\text { metodologia experimental e a preocupação com o comportamento, não } \\
\text { com a mente". }\end{array}$ & 2 \\
\hline
\end{tabular}

A leitura dos trechos destacados no quadro com as citações de Bandura permite perceber que o autor foi inserido nos artigos dos autores brasileiros a partir da abordagem de conceitos centrais da TSC. No artigo de 1981, Borges discute dois conceitos primários: o operante e o conceito de aprendizagem social. Faz-se a distinção entre a aprendizagem por reforçamento de Skinner e a aprendizagem social de Bandura Vê-se que nesse texto o autor do artigo está focado em uma das teorias bandurianas, a teoria da aprendizagem social, referência de vários trabalhos desse autor e seus colaboradores, em uma linha informativa e explicativa do processo comportamental da aprendizagem por observação

No artigo de 1983, Kerbauy apresenta as terapias comportamental e cognitiva comportamental como distintas, referindo-se ao fato de a terapia comportamental cognitiva aceitar elementos mediadores como crenças, percepções e pensamentos. Refere-se à obra de Bandura de 1969, entre outros autores, que estão sendo identificados no texto como autores cognitivos.

No artigo de 1998, Seminério faz referência expressa à importância do trabalho de Bandura para conter a disseminação de violência e agressividade junto à juventude. Nesse artigo cita Bandura como fonte de reafirmação de uma explicação para fenômeno social.

No artigo de 1999, Simonassi, buscando o que faz solucionar-se problemas, refere-se ao conceito de "consciência" apresentado por Bandura em sua obra de 1979, em que explícita a função das atividades simbólicas nas ações, pensamentos e emoções. A discussão da cognição na perspectiva de Bandura, em seu livro de 1969, é o foco dos destaques neste artigo. No segundo artigo de 1999, De Rose destaca a cognição como a razão da citação de Bandura na medida em que é por ela que o autor delimita o bloco comportamentalista. Além disso, apresenta autores com trabalhos de grande relevância e qualidade que se aproximam ou divergem das ideias de Skinner. Considera Bandura um pesquisador com uma produção de alta qualidade/relevância com maior grau de divergência do pensamento de Skinner.

No que diz respeito a temas, é possível perceber que Borges articula seu texto para o tema do planejamento instrucional, Kerbauy para a terapia, Seminério para a discussão de religião e Simonassi e de Rose para os aspectos mais teóricos.

Até aqui foram explorados com algumas análises qualitativas apenas os cinco artigos que citaram Bandura antes da década de 2000. Movimento equivalente, incluindo a identificação dos textos citados nos artigos para as demais ocorrências observadas nos 163 artigos, já está sendo realizado pois o levantamento artigo a artigo ainda está em andamento.

Os resultados apresentados neste artigo confirmam a hipótese inicial de que o pensamento banduriano demorou a ser identificado no cenário brasileiro como fonte de interlocução dos pesquisadores que publicaram nos periódicos analisados. Ainda que tenham sido localizados dois trabalhos da década de 1980, eles não foram acompanhados de outros que revelassem que o pensamento de Bandura estivesse em rota de firmar-se entre as referências brasileiras. A hipótese levantada pelas autoras para o projeto-mãe, 
em que esta discussão acontece considera a crescente e forte presença da Análise do Comportamento desde os idos da década de 1960 como contexto desfavorecedor do pensamento de Bandura pela Teoria da Aprendizagem Social. Tal assertiva funda-se na publicação do livro "Modificação de Comportamento" que já então apontava a trilha da cognição em desbravamento por aquele autor. Apesar das pistas que este artigo mostra a este respeito, há que se explorar futuramente os dados que estão sendo levantados sobre o assunto nos diversos artigos localizados por este trabalho.
Em busca de respostas para outras questões formuladas para o projeto maior do qual este artigo é parte, a escrita de novos textos está em andamento, agora explorando as áreas em que foram localizadas citações de Bandura, quais foram as obras citadas, os constructos abordados, a interlocução deles com outros autores, entre outras. Acredita-se que, com este movimento complementar, será possível construir um completo panorama das condições em que o pensamento banduriano foi abordado nos artigos de periódicos brasileiros.

\section{Referências}

Azzi, R. G. (2014). Introdução à teoria social cognitiva (Série teoria social cognitiva em contexto educativo, vol 1). São Paulo, SP: Casapsi.

Azzi, R. G., Polydoro, S. A. (Orgs.). (2006). Autoeficácia em diferentes contextos. Campinas, SP: Alínea.

Bandura, A. (1986). Social foundations of thought and action: A social cognitive theory. Englewood Cliffs, NJ: Prentice-Hall.

Bandura, A. (1997). Self-efficacy: The exercise of control. New York, NY: Freeman.

Brozek, J. (1998). Abordagem quantitativa: Wundt na América. In J. Brozek, \& M. Massimi, Historiografia da psicologia moderna. São Paulo, SP: Loyola.

Diener, E., Oishi, S., \& Park, J. (2014). An incomplete list of eminent psychologists of the modern era. Archives of Scientific Psychology, 2(1), 20-31. https://doi.org/10.1037/arc0000006

Dittimann, M. (2002). Study ranks the top 20th century psychologists. Monitor on Psychology, 33(7), 28-29.

Ferreira, L. C. M. (2003). A pesquisa sobre a habilidade de leitura, em classes regulares, no Journal of Appled Behavior Analysis de 1968 a 2002 (Dissertação mestrado). Pontifícia Universidade Católica de São Paulo, São Paulo, SP, Brasil.

Ferreira, L.C.M., Guedes, M.C., \& Azzi, R.G. (2004) Análise do comportamento e a habilidade de leitura: Um levantamento crítico de artigos do JABA. PsicoUSF, 9(1), 59-69.

Green, E. (2016, maio 12). What are the most-cited publications in the social science (according to Google scholar)? LSE Impact Blog.

Narin, F, Olivastro, D., Stevens, K. S. (1994). Bibliometric theory, practive and problem. Evaluation Review, 18(1), 65-76. https:// doi.org/10.1177/0193841X9401800107

Parrat-Dayan, S. (2008) A recepção da obra de Piaget nomeio pedagógico dos anos 1920-1930. Schème: Revista Eletrônica de Psicologia e Epistemologia Genética, 1(2), 148-167. https://doi.org/10.5555/repeg.vli2.568

\section{Roberta Gurgel Azzi}

Doutora em Educação pela Universidade Estadual de Campinas, Campinas - SP. Brasil. Diretora da TSC - Centro de Estudos e Pesquisas, Bragança Paulista - SP. Brasil.

E-mail: azzi.tsc@gmail.com

(iD) https:// orcid.org/0000-0003-0971-7852

\section{Ana Paula Basqueira}

Doutora. Docente da Anhanguera Educacional. Campinas - SP. Brasil.

E-mail: ana.basqueira@anhanguera.com

(iD) https:// orcid.org/0000-0001-6580-5676 


\section{Luiza Cristina Mauad Ferreira}

Doutorado em Educação na área de concentração Psicologia, Desenvolvimento Humano e Educação pela Universidade Estadual de Campinas, Campinas - SP. Brasil. Diretora geral da B\&G Assessoria Educacional e de Saúde, Itajubá-MG. Brasil.

E-mail: luizacmauad@hotmail.com

(iD https://orcid.org/0000-0003-2960-8784

\section{Maria do Carmo Guedes}

Doutora pela Pontifícia Universidade Católica de São Paulo (PUC-SP), São Paulo - SP. Brasil.

E-mail: mcguedes34@gmail.com

(iD https://orcid.org/0000-0003-4062-3694

Mônica Helena Tieppo Alves Gianfaldoni

Doutora pela Pontifícia Universidade Católica de São Paulo (PUC-SP), São Paulo - SP. Brasil.

E-mail:mhtag@pucsp.br

(iD https://orcid.org/0000-0002-8689-8469

Endereço para envio de correspondência:

B\&G Assessoria em Educação e Saúde. Rua Francisco Masseli, 966. CEP: 37500-058. Itajubá - MG. Brasil.

Recebido 07/11/2017

Reformulado 17/04/2018

Aceito 25/06/2018

Received 11/07/2017

Reformulated 04/17/2018

Approved 06/25/2018

Recibido 07/11/2017

Reformulado 17/04/2018

Aceptado 25/06/2018

Como citar: Azzi, R. G., Ferreira, L. C M., Basqueira, A. P., Guedes, M. do C. \& Gianfaldoni, M. H. T. A. (2019). Citações de Obras de Bandura em Artigos de Periódicos de Psicologia Brasileiros: Uma Análise Preliminar. Psicologia: Ciência e Profissão, 39, 1-14. https://doi.org/10.1590/1982-3703003187 551

How to cite: Azzi, R. G., Ferreira, L. C M., Basqueira, A. P., Guedes, M. do C. \& Gianfaldoni, M. H. T. A. (2019). Citations of Bandura's Works in Articles of Brazilian Psychology Journals: a Preliminary Analysis. Psicologia: Ciência e Profissão, 39, 1-14. https://doi.org/10.1590/1982-3703003187551

Cómo citar: Azzi, R. G., Ferreira, L. C M., Basqueira, A. P., Guedes, M. do C. \& Gianfaldoni, M. H. T. A. (2019). Citas de Obras de Bandura en Artículos de Periódicos de Psicología Brasileños: Un Análisis Preliminar. Psicologia: Ciência e Profissão, 39, 1-14. https://doi.org/10.1590/1982-3703003187551 